\title{
Delivering Complex Care: Designing for Patients and Physicians
}

\author{
Jeremy A. Epstein, MD (1) and Albert W. Wu, MD \\ Department of Medicine, General Internal Medicine, Johns Hopkins University School of Medicine, Baltimore, MD, USA.
}

The management of high-utilizing patients is an area of active research with broad implications for the healthcare system. There are significant operational challenges to designing primary care models for these medically complex, high-needs patients. Although it is crucial to provide a high degree of continuity of care for this population, managing a cohort of these patients can lead to provider over-work and attrition. This may be magnified by the lack of training dedicated to addressing the unique care needs of these patients. While academic medical centers would seem well suited to care for individuals with multimorbidity needing intensive and specialized treatment, primary care providers in this setting need additional support to be clinically available for patients while pursuing scholarship and teaching. Formally recognizing intensive outpatient care as a specialty within internal medicine would help overcome some of these challenges. This would require a committed effort to high-level systems changes including a new focus on graduate medical education, the creation of division-level infrastructure within academic departments of medicine, and realistic levels of financial support to make this a viable career path.

$\mathrm{J}$ Gen Intern Med 36(3):772-4

DOI: $10.1007 / \mathrm{s} 11606-020-06212-3$

(C) Society of General Internal Medicine 2020

I n healthcare, "high utilizers" are complex patients who use a disproportionate amount of health care relative to their peers. The burden of repeated episodes of illness and treatment falls heavily on these people. Much of this cycle seems avoidable, and in the last decade, attempts to improve care for these patients have gained momentum. ${ }^{1,2}$ The most promising interventions have involved rethinking the roles of providers and the practices that care for these patients. ${ }^{3-5}$

Complex patients benefit most from a high degree of continuity of care and attention from their primary care providers. $^{6-13}$ However, maximizing provider accessibility to match the needs of this population places heavy demands on the time and availability on those clinicians. So, to do it, the

Prior Presentations None.

Received April 13, 2020

Accepted September 3, 2020

Published online September 15, 2020 medical community must grapple with what can or should be expected from the modern physician. In this commentary, we discuss the challenges in designing practice models that optimize care for high-utilizing patients.

When designing a care model for complex patients, one of the primary questions is who will deliver that care. One requirement is that the model has appropriate structure and support to maintain a high degree of patient-provider continuity. This produces physicians who are intimately familiar with their patients, aware of their social and medical challenges, and up to date with their past and current care. Greater continuity of care is associated with decreased hospitalization, better patient care metrics, and lower costs. ${ }^{13}$ This stands in contrast to fragmented care, which is inefficient, and leads to unnecessary repeat testing, broken handoffs, misunderstandings, and patient dissatisfaction. ${ }^{14}$ However, while being the single doctor responsible for a cohort of chronically ill and needy patients can generate a high degree of patient-provider continuity, it may be a prescription for burnout. ${ }^{15,16}$ Physician well-being and burnout have become topical, with national multidisciplinary organizations (National Academy of Medicine: Action Collaborative on Clinician Well-Being and Resilience) and recent medical conferences (American Conference on Physician Health 2019, American Academy of Family Physicians 2020) focused squarely on these issues. We have a nostalgic impression of the always-available full-time internist as an enviable position that commanded respect. However, today, few physicians would be likely to sign up for this job description. To be sustainable, the position of a complex patient care provider needs to be crafted to avoid burn out and attrition.

Primary care physicians within academic medical centers would seem well positioned to manage patients with multimorbidity given their need for specialty care and interdisciplinary coordination. ${ }^{17,} 18$ However, designing this type of practice for an academic environment poses specific challenges. Physicians at academic centers are already keenly aware of the need to juggle clinical responsibilities with scholarship and teaching - serving the faculty mission of advancing medical knowledge through education and research. At many academic centers, a strictly clinical focus may not provide a viable path to promotion, which requires research and publications and a national or international reputation in the physician's field. Maintaining this balance may be all the more difficult when serving as an intensive outpatient physician. 
Ensuring continuity of care for complex patients requires a large clinical footprint with a focus on this high-risk cohort. Yet, without robust coverage systems that adequately serve the needs of these medically complex patients, time otherwise apportioned to research may be interrupted by patient care. With the success of intensive outpatient programs often being measured by decreases in medical expenses and reductions in emergency room visits or admissions, physicians feel pressured to stop other activities to personally oversee patient care. A final barrier for academic physicians in this care model is the lack of education about complex patient care in the current medical curriculum and a corresponding lack of comfort undertaking this role. Few residency programs have dedicated training on how to care for complex patients with frequent hospitalizations. In fact, these patients are often shunted away from residency primary care clinics to full-time physicians to help improve continuity, which is de facto limited by the threeyear duration of residency.

Perhaps what is needed is to formally recognize the position of outpatient intensivist. To support these new subspecialists, there needs to be a fundamental shift in the structure and value placed on this field. Patients who occupy the apex of healthcare utilization and suffer from complex multimorbidity have unique needs, requiring providers with specialized skills practicing in distinctive care models. Intensive primary care therefore merits a new framework that is rooted in general internal medicine but extends beyond. To start, intensive outpatient care needs to be integrated with, rather than separated from, residency training. As with other medical specialties, fellowships may be needed to train residents to become competent outpatient intensivists. At the institutional level, recognizing intensive outpatient care as a formal division of internal medicine, and affording it a commensurate level of support, would make it possible to recruit and retrain skilled academic physicians in this field. In recognition of the high potential for burnout, there would need to be a significant reduction in patient panel sizes to offset the increased needs and clinical demands. This would need to be paired with a promotional pathway that recognizes the value of this kind of care. At the national level, the current reimbursement levels are insufficient to cover the time and resources that would be required. Adjustments would be needed in the current payment scheme to raise compensation accordingly.

In summary, our healthcare system is faced with the ongoing need to care for a growing group of complex, high-utilizing, chronically ill patients. Academic medical centers could design a new subspecialty of outpatient intensivists to take on this challenge. While we have focused on primary care physicians, we acknowledge that there are other models and types of providers, such as community health centers, team-based care, and nurse practitioners, that care for complex patients. There is still much to learn about the effectiveness of these alternatives and how physician-centered outpatient care would fit into this ecosystem, to both complement and coordinate with other providers. For example, a recent publication showed that even the Camden Coalition's capable multidisciplinary teams of nurses, social workers, and community health workers failed to reduce readmissions amongst high-utilizing patients. ${ }^{19}$ Research to date suggests that outpatient intensivist care would at a minimum allow complex patients substantially more time for ambulatory visits, and a detailed curriculum devoted to addressing social determinants of health. ${ }^{20,21}$ However, a committed effort to high-level systems change is needed including establishing curricula for medical students and medical residents surrounding the care of patients with multimorbidity and crafting a framework for formal specialty training and its certification parameters. This new focus on graduate medical education would need to be paired with the creation of division-level infrastructure within academic departments of medicine and realistic levels of financial support to make this a viable career path. Until such changes are made, intensive outpatient clinicians may continue to struggle to contend with medical, professional, and personal barriers that conflict with what is best for their patients.

Corresponding Author: Jeremy A. Epstein, MD; Department of Medicine, General Internal Medicine, Johns Hopkins University School of Medicine, Baltimore, MD, USA (e-mail: Epstein@jhmi.edu).

\section{Compliance with Ethical Standards:}

Conflict of Interest: The authors have no conflicts of interest to declare.

\section{REFERENCES}

1. Baker JM, Grant RW, Gopalan A. A systematic review of care management interventions targeting multimorbidity and high care utilization. BMC Health Serv Res. 2018;18(1):65.

2. Edwards ST, Peterson K, Chan B, Anderson J, Helfand M. Effectiveness of Intensive Primary Care Interventions: A Systematic Review. J GEN INTERN MED. 2017;32(12):1377-1386.

3. Sander LD, Albert M, Okeke N, et al. Building a Medicaid Ambulatory Complex Care Program Within an Urban Medical Home. Popul Health Manag. 2018.

4. Yoon J, Chang E, Rubenstein LV, et al. Impact of primary care intensive management on high-risk veterans' costs and utilization: A randomized quality improvement trial. Ann Intern Med. 2018.

5. Anderson GF, Ballreich $\mathbf{J}$, Bleich $\mathbf{S}$, et al. Attributes common to programs that successfully treat high-need, high-cost individuals. Am J Manag Care. 2015;21(11):e597-600.

6. Maciejewski ML, Powers BJ, Sanders LL, et al. The intersection of patient complexity, prescriber continuity and acute care utilization. J GEN INTERN MED. 2014;29(4):594-601.

7. Romaire MA, Haber SG, Wensky SG, McCall N. Primary care and specialty providers: an assessment of continuity of care, utilization, and expenditures. Medical care. 2014;52(12):1042-1049.

8. Hussey PS, Schneider EC, Rudin RS, Fox DS, Lai J, Pollack CE. Continuity and the costs of care for chronic disease. JAMA Intern Med. 2014;174(5):742-748.

9. Cheng S-H, Chen C-C, Hou Y-F. A longitudinal examination of continuity of care and avoidable hospitalization: evidence from a universal coverage health care system. Arch Intern Med. 2010;170(18):1671-1677.

10. Bazemore A, Petterson S, Peterson LE, Bruno R, Chung Y, Phillips RL, Jr. Higher Primary Care Physician Continuity is Associated With 
Lower Costs and Hospitalizations. Annals of family medicine. 2018;16(6):492-497.

11. Arthur KC, Mangione-Smith R, Burkhart Q, et al. Quality of Care for Children With Medical Complexity: An Analysis of Continuity of Care as a Potential Quality Indicator. Acad Pediatr. 2018;18(6):669-676.

12. Amjad H, Carmichael D, Austin AM, Chang C-H, Bynum JPW. Continuity of Care and Health Care Utilization in Older Adults With Dementia in Fee-for-Service Medicare. JAMA Intern Med. 2016;176(9): 1371-1378.

13. Walker J, Payne B, Clemans-Taylor BL, Snyder ED. Continuity of Care in Resident Outpatient Clinics: A Scoping Review of the Literature. Journal of graduate medical education. 2018;10(1):16-25.

14. Bodenheimer T. Coordinating Care - A Perilous Journey through the Health Care System. New England Journal of Medicine. 2008;358(10):1064-1071.

15. Pedersen AF, Nørøxe KB, Vedsted P. Influence of patient multimorbidity on GP burnout: a survey and register-based study in Danish general practice. Br J Gen Pract. 2020;70(691):e95-e101.

16. Helfrich CD, Simonetti JA, Clinton WL, et al. The Association of Team-Specific Workload and Staffing with Odds of Burnout Among
VA Primary Care Team Members. J GEN INTERN MED. 2017;32(7):760-766.

17. Komaromy M, Bartlett J, Zurawski A, et al. ECHO Care: Providing Multidisciplinary Specialty Expertise to Support the Care of Complex Patients. J GEN INTERN MED. 2020;35(1):326-330.

18. Loeb DF, Bayliss EA, Candrian C, de Gruy FV, Binswanger IA. Primary care providers' experiences caring for complex patients in primary care: a qualitative study. BMC Family Practice. 2016;17(1):34.

19. Finkelstein A, Zhou A, Taubman S, Doyle J. Health Care Hotspotting A Randomized, Controlled Trial. New England Journal of Medicine. 2020;382(2):152-162.

20. Meltzer DO, Ruhnke GW. Redesigning care for patients at increased hospitalization risk: the Comprehensive Care Physician model. Health Aff (Millwood). 2014;33(5):770-777.

21. Paul SA. The value of primary care. In. The University of Chicago Magazine. Vol 111. Chicago, IL 2019:40-47.

Publisher's Note: Springer Nature remains neutral with regard to jurisdictional claims in published maps and institutional affiliations. 